\title{
Theoretical and Experimental Study of the Mechanism of Reaction Formation of Cyanine Dyes
}

\author{
Boshkayeva Assyl, Omarova Roza, Pichkhadze Guram, Shalpykova Nasiba \\ Asfendiyarov Kazakh National Medical University, Almaty, Kazakhstan \\ Email: kenes65@mail.ru
}

Received 30 September 2014; revised 17 November 2014; accepted 3 December 2014

Copyright (C) 2014 by authors and Scientific Research Publishing Inc.

This work is licensed under the Creative Commons Attribution International License (CC BY). http://creativecommons.org/licenses/by/4.0/

(c) () Open Access

\section{Abstract}

To study the theoretical bases of the mechanism of reaction formation of cyanine dyes, special importance is gained by methods of quantum chemistry. The use of these methods is provided with the known molecular and dynamic HyperChem program. The purpose of studying was quantum and chemical studying of features of a geometrical and electronic structure of model molecules of penicillin acid, of derivative of glutaconic dialdehyde (DGD), and the cyanine dyes, and also an assessment of power of process of their formation. For studying was carried out the experimental mark of chemism of course of reaction with theoretical justification of the mechanism of reaction formation of cyanine dyes. The results show that all studied model molecules are thermodynamic steady systems to what values of enthalpies of their formation, rather high on an absolute value, testify negative on a sign. Reaction goes by the mechanism of nucleophilic addition.

\section{Keywords}

Cyanine Dye, $\beta$-Lactam, Quantum-Chemical Method, Penicillin Acid, Derivative of Glutaconic Dialdehyde, Electronic Density, The Binding Energy, HyperChem

\section{Introduction}

As is known, the proximity of chemical structure, similarity methods of obtaining and the presence of certain structural groupings of cyanine dyes form the basic principles of their classification. According to the standard classification of dyes, based on the principle of generality of chromorphic systems, cyanines are organic compounds containing two hetero-cyclic residues connected by chain of methine groups. In organic chemistry, chromorphic system of cyanine dyes consists of a chain of free or substituted methine groups with electron-donors 
and electron-acceptor substituents at the ends; where in the substituents and a part of methine groups may be a part of the aromatic or heterocyclic radicals, [1]-[9]. Cyanine dyes are formed by reaction of either the opening of the pyridine cycle, pyrimidine and furan rings, or when some condensation reactions.

One of the proposed varieties of using condensation reaction types in the analysis of drugs is the reaction of forming the cyanine dyes. The reaction is based on a combination of derivative of glutaconic dialdehyde (DGD), as one of the cleavage products of the pyridine ring, with the products of hydrolytic cleavage of $\beta$-lactam. Theoretical grounding of the reaction mechanism is considered from the perspective of existing classical electronic effects.

One of the formed acid hydrolysis products of penicillin salts reacts with DGD. Unlike the thiazolidine ring, $\beta$-lactam ring of penicillin is readily cleaved under the action of alkalis, acids and other substances. This explains, for example, the formation of cleavage products of $\beta$-lactam, [10]. Features of the molecular structure of penicillin may be the cause of its behavior in the presence of various reagents. Under the action of $25 \%$ hydrochloric acid, penicillins are inactivated with forming corresponding penillic and penicillin acids [11] [12].

Based on the structural features of $\beta$-lactam, the appearance of coloring reaction product with DGD facilitate: a chain of alternating single and double bonds (with the chain involved in a double bond between carbon and nitrogen); the presence of groups or atoms strongly attracting electrons to the overall electronic system of the molecule; planar arrangement of atoms. Some fragments in molecule of benzylpenicillin sodium leave the plane, but they do not affect the structural changes which cause the appearance coloring cyanine dyes. Carbon atoms chain bounded to each other by alternating single and double bonds has a direct value for the structure of the colored compound. Expected colored compound in this reaction is subjected to selective absorption of light energy with wavelengths in the range of the visible spectrum.

Electronic density redistribution in penicillin acid molecule as one of the products of hydrolytic cleavage of penicillin has a significance for revealing reaction capacity of $\beta$-lactam, son a qualitative level.

The study of reaction formation of cyanine dyes has given confirmation of condensation reaction of DGD with penicillin acid based on the selected conditions of this reaction. Effect of substituents on the electronic density distribution in the molecule of penicillin acid was evaluated by electronic effects-inductive (I) and mesomeric (M). In the last molecule, the electron density distribution is considered (Figure 1). The direction of shift of electronic density is designated by a curved arrow.

In penicillin acid molecule, a reactive center is nitrogen of an iminogroup having electronegative character (electronegativity of nitrogen-3.0) as compared with sp2-hybridized carbon atom (carbon electronegativity-2.8). Due to the lone pairs of electrons, nitrogen may bind electrophilic reagent. Due to nitrogen of iminogroup (-I), inductive effect is determined. As a result of its action, penicillin acid molecule may have a deficiency and of electronic density on the nitrogen of an iminogroup-its excess. NH has electron donor impact somewhat increasing the electron density in the molecule due to p, p-conjugation ( $+\mathrm{M}$ effect). It is also noted that when looking at the colors, depending on the state of the electrons in the molecule of penicillin acid, p-electrons displacement occurs along the entire system of conjugated double bonds in the molecule. As described above, the mobility of p-electrons relates to the connection between two carbon atoms and those structures where there are p-bond between carbon and nitrogen.

Thus, the transition of the molecule in the excited state, the polarization of the molecule, the mobility of pelectrons, produce of continuous distribution of positive and negative charges in the molecule are responsible for the appearance of coloring for condensation product DGD with penicillin acid.

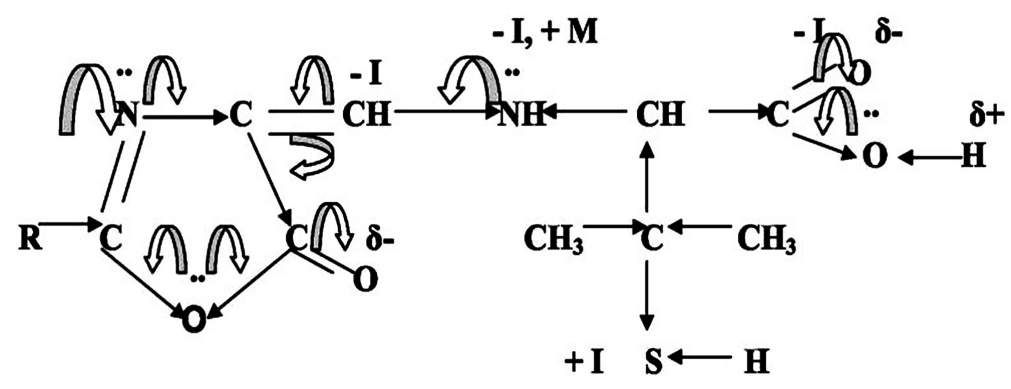

Figure 1. The electronic density distribution in the molecule of penicillin acid. 


\section{Materials and Methods}

The used methods are: Portable HyperChem 8.0.7, MOPAC-packages of quantum chemical programs. In this paper we used the PM3-parametric method 3 (a semiempirical approach), which is a version of the AM1 method and differs from this method by parameter values only. Parameters for PM3 were obtained by comparing the number and type of experiments with the results of calculations.

\section{Results and Discussion}

According to theoretical calculations, we have proven that the interaction of penicillin acid with pyridinium salt (the rodanopiridiny chloride formed at action on pyridine of the splitting reagents (chloroamine B and thiocyanate of ammonium)) in the first stage reaction there occurs nucleophilic addition of mesomeric anion (nitrogen of secondary amino group) to the resulting rodanopiridinium chloride. In the stage of formation of cyanine dyes there occurs splitting of cycle pyridine diethylamide of nicotinic acid (DENA) to form DGD, which in third stage is condensed with a molecule of penicillin acid (product absorbing in the visible region at a wavelength $\lambda_{\max }=565 \mathrm{~nm}$ is formed).

Conjugation of secondary amino group nitrogen of penicillin acid to rodanopiridiniumchloride contributes to dynamic factor determining the stability of intermediate species formed during the reaction products (there are particles with extended conjugated systems, and it is known that coupling stabilizes not only the molecules, but also the intermediate particles, and this is what determines the ease of formation of the intermediate particles and determines the entire course of the reaction). The reaction takes place only in the direction that provides the energy gain. Product formed during the reaction, staining in purple, is a molecule with a very large conjugated system.

In practical experiments, the study of the absorption spectra of DGD in the UV region shows that in the acidic environment a colorless form glutaconic dialdehyde is formed. In weak acidic medium with the cleavage of $25 \%$, $6 \%$ of DENA solution yellow enol form of glutaconic dialdehyde is formed. In an unstable condition yellow enol form becomes red monoanilid of glutaconic dialdehyde, on which is drawn attention on reaching the optimal reaction conditions of forming cyanine dyes. The reaction proceeds in a stoichiometric ratio between the penicillin acid and derivative of glutaconic dialdehyde (DGD). Penicillin acid following acid hydrolysis of benzylpenicillin sodium salt is combined with the DGD, which is formed by splitting the pyridine cycle of diethyl-nicotinic acid under the action of thiocyanate chloride at $\mathrm{pH} 2.0$ - 3.0. The last one previously prepared by interaction of chloramine B and thiocyanate ammonium. Hydrolysis of penicillin and cephalosporin antibiotics is performed by using aqueous solutions of hydrochloric acid.

Penicillin and cephalosporin antibiotics undergo hydrolytic degradation for 5 - 10 minutes at a water bath temperature $\left(98^{\circ}\right)$. Establishing a phased sequence of this reaction with the selection of all the conditions and explanation of one of the theoretical versions of the reaction mechanism of nucleophilic conjugationis given in the papers [13] [14].

In general, chemical reactions can be traced to the relationship between the yield of the final product and the indices of reaction ability of the reactants (atomic charges, bond orders, energy of frontier molecular orbitals (MO), the squares of the coefficients of the expansion of frontier MOs by the basis of atomic orbitals (AO) etc.) [15]-[18]. Charges on the atoms and the parameters of frontier MOs are mostly often used. The yield of the final reaction product is determined by the free energy activation, and the indices of reaction ability characterize the energy of the intermolecular interactions of the reagents. However, for certain reactions, these parameters are linked to each other. For example, with an increase in the interaction energy between the reagents its free energy of activation decreases.

Intermolecular interaction energy during the approach of reagents can be divided into three types of contributions: coulon, orbital and steric. Coulon interaction energy depends on the electron density distribution, or the charges on the atoms of the reagents. Therefore, some reactions can succeed finding in a relationship between the charges on the atoms and yield of the final reaction products. Thus, nucleophilic reagents (attacking center is negatively charged) joined mainly to atoms, which are localized by large positive charges and electrophilic (attacking center is positively charged), on the contrary-to atoms, which are localized by large negative charges.

Orbit interaction of any MO pairs is inversely proportional to difference of their energies, i.e. the further apart are the orbitals on scale of energies, the less they interact. Therefore, in practice, we usually use the approach of the frontier orbitals. In this approximation, the energy of the orbital interaction depends on the energy of frontier 
MO and the coefficients of these MO decomposition by the AO basis. Any of these values can be used as an index of reactivity, but the energy difference of frontier MO is most lyused. Furthermore, the shape of frontier MO allows to make conclusions about the mechanisms of organic reactions as electrophilic attack often comes in the highest values of the highest occupied MO (HOMO) and nucleophilic-in the place of the greatest values of the lowest unoccupied MO (LUMO).

As a result, of molecular modeling of substances molecules entering into the reaction, and the molecules of the cyanine dye with using quantum-chemical method AM1 there were obtained data, confirming that the reaction can go by the mechanism of nucleophilic addition. More precisely, due to the distribution of electronic density in the molecules of DGD and penicillin acid, it can be said that a negatively charged nitrogen atom in the molecule penicillin acid may attack the positively charged carbon atom in the strongly polarized carbonyl group of DGD. In addition, the LUMO energy of penicillin acid is positive, therefore, this molecule is nucleophilic.

The electron density distribution in the molecule of penicillin acid is shown in Figure 2.

The electronic density distribution in it is shown in Figure 3.

The optimized geometry of the cyanine dye molecule is shown in Figure 4.

Geometric and electronic parameters of the objects under study are presented in Tables 1-3.

The analysis of the results in Tables 1-3 follows that the coupling reaction of penicillin acids with DGD with forming cyanine dyes occurs by the interaction of the positively charged carbon of DGD with a free electron pair of a nucleophilic center, i.e. negatively charged nitrogen atoms in the molecule of penicillin acid.

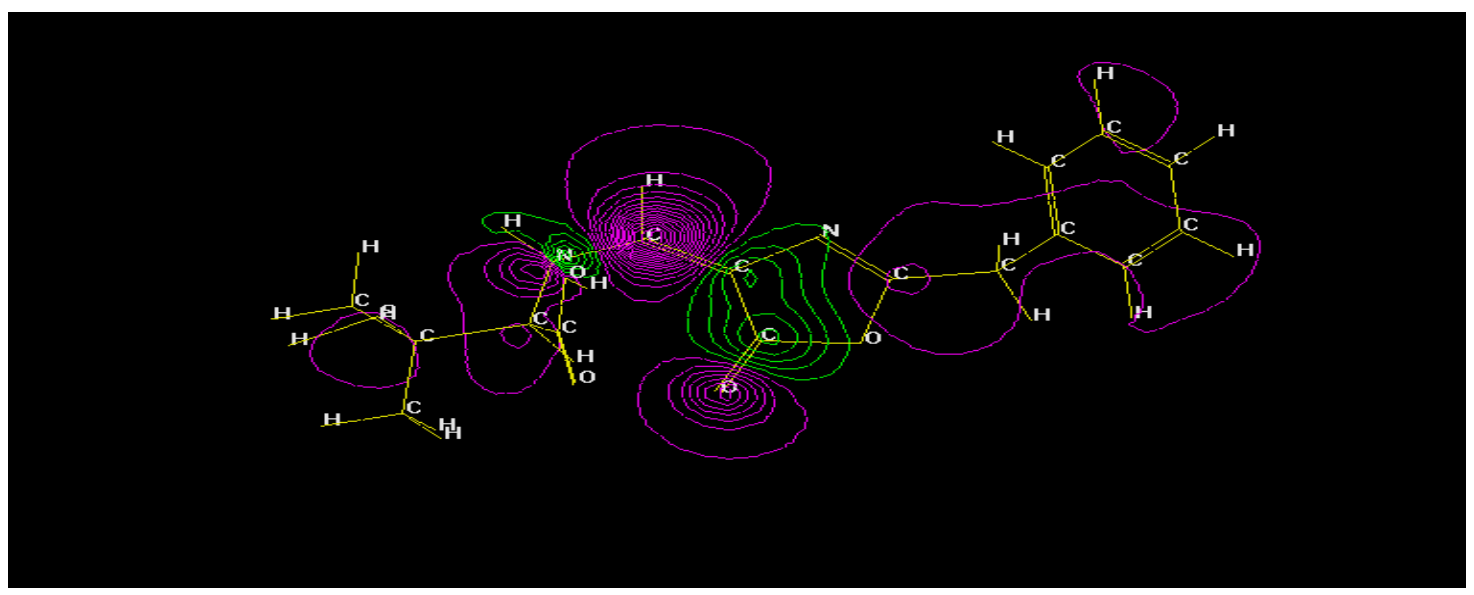

Figure 2. Distribution of the electronic density in the optimized molecule of penicillin acid $(\mathrm{ENSMO}=1.69 \mathrm{eV})$.

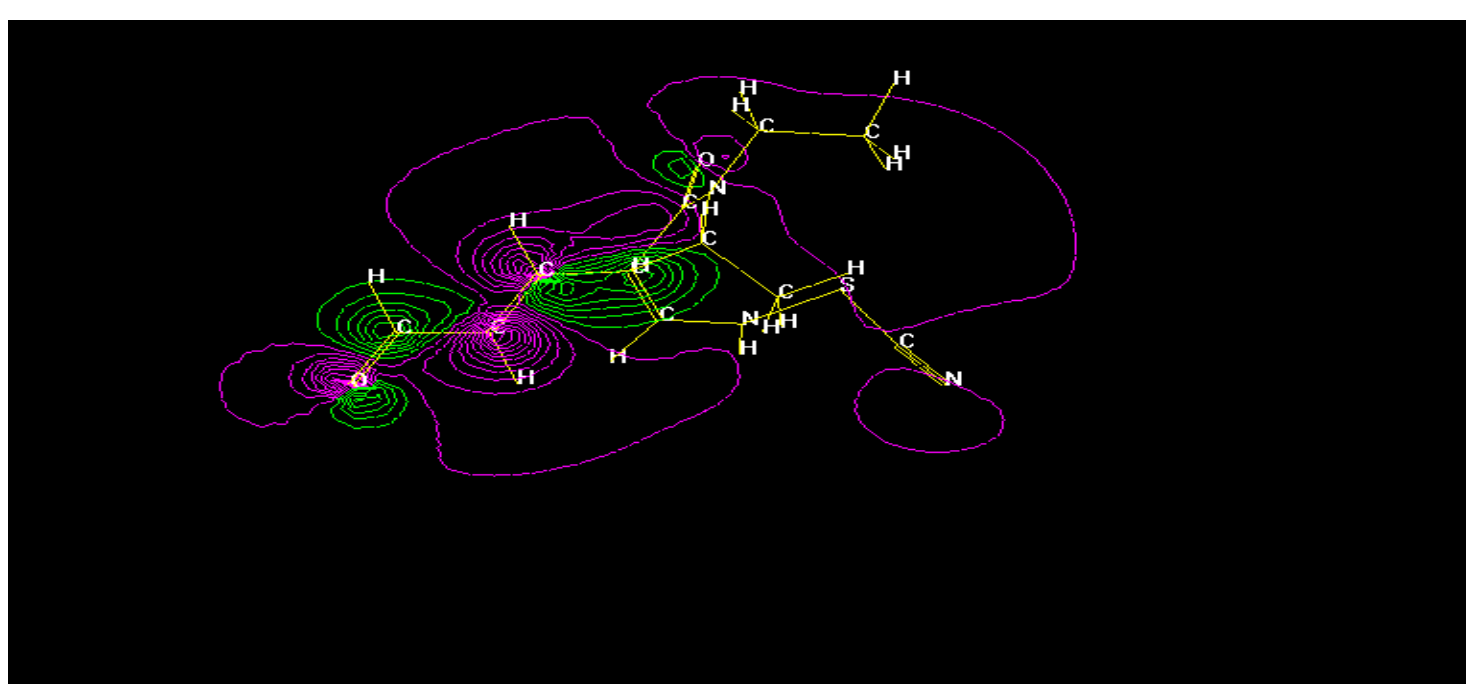

Figure 3. The distribution of electronic density in the optimized molecule of DGD $($ ENSMO $=-1.07 \mathrm{eV})$. 


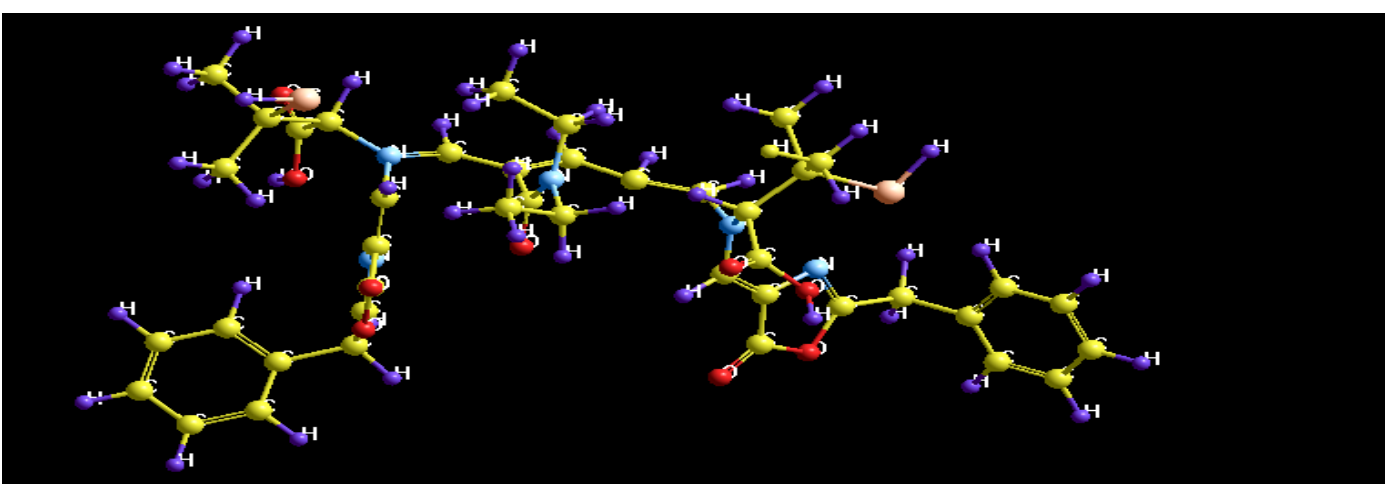

Figure 4. Geometric model of cyanine dye molecule.

Table 1. Geometric baseline parameters, reacting agents and final reaction products.

\begin{tabular}{|c|c|c|c|c|}
\hline \multicolumn{2}{|c|}{ Geometric parameters } & Penicillin acid & DGD & Cyanine dye \\
\hline \multicolumn{2}{|c|}{ Enthalpy of formation ( $\Delta \mathrm{N} 0 \mathrm{obr})$} & $\begin{array}{c}-84.2679 \mathrm{kcal} / \mathrm{mol} \\
(-352.8 \mathrm{KJ} / \mathrm{mol})\end{array}$ & $\begin{array}{c}-7.8852276 \mathrm{kcal} / \mathrm{mol} \\
(-3.01 \mathrm{KJ} / \mathrm{mol})\end{array}$ & $\begin{array}{c}-129.631 \mathrm{kcal} / \mathrm{mol} \\
(-542 \mathrm{KJ} / \mathrm{mol})\end{array}$ \\
\hline \multicolumn{2}{|c|}{ Dipole moment $(\mu)$} & $3.571 \mathrm{D}$ & $0.953 \mathrm{D}$ & $5.145 \mathrm{D}$ \\
\hline \multirow{2}{*}{$\begin{array}{l}\text { Energy of } \\
\text { frontier orbitals }\end{array}$} & $\begin{array}{l}\text { Energy of LUMO } \\
\text { (lowest unoccupied } \\
\text { molecular orbital) }\end{array}$ & 1.699999 & -1.070426 & -- \\
\hline & $\begin{array}{l}\text { Energy of the HOMO } \\
\text { (highest occupied } \\
\text { molecular orbital) }\end{array}$ & -9.739995 & -9.370866 & -- \\
\hline
\end{tabular}

Table 2. Electronic parameters of the molecules of penicillin acid and DGD.

\begin{tabular}{|c|c|c|c|}
\hline Parameter & Penicillin acid & Parameter & DGD \\
\hline$\mu, D$ & 3.571 & $\mu, D$ & 0.953 \\
\hline $\mathrm{qC}_{1}$ & -0.121483 & $\mathrm{qC}_{1}$ & 0.210 \\
\hline $\mathrm{qC}_{2}$ & -0.131220 & $\underline{\mathbf{q} \mathbf{O}_{2}}$ & $\underline{-0.284}$ \\
\hline $\mathrm{qC}_{3}$ & -0.130831 & $\mathrm{qC}_{3}$ & -0.270 \\
\hline $\mathrm{q} \mathrm{C}_{4}$ & -0.112560 & $\mathrm{q} \mathrm{C}_{4}$ & -0.030 \\
\hline $\mathrm{qC}_{5}$ & -0.086901 & $\underline{\mathbf{q} \mathbf{C}_{5}}$ & $\underline{-0.199}$ \\
\hline $\mathrm{qC}_{6}$ & -0.110834 & $\mathrm{q} \mathrm{C}_{6}$ & 0.05 \\
\hline $\mathrm{q} \mathrm{C}_{11}$ & -0.057496 & q N7 & -0.469 \\
\hline $\mathrm{q} \mathrm{C}_{14}$ & 0.082292 & q S13 & 0.620 \\
\hline q $\mathrm{N}_{16}$ & -0.153666 & q C14 & -0.429 \\
\hline $\mathrm{q} \mathrm{O}_{17}$ & -0.226165 & q N15 & 0.005 \\
\hline $\mathrm{q} \mathrm{C}_{18}$ & -0.198795 & $\mathrm{q} \mathrm{C}_{16}$ & 0.351 \\
\hline $\mathrm{q} \mathrm{C}_{19}$ & 0.341160 & $\mathrm{q} \mathrm{O}_{17}$ & -0.361 \\
\hline $\mathrm{q} \mathrm{O}_{20}$ & -0.293519 & $\mathrm{q} \mathrm{N}_{18}$ & -0.322 \\
\hline $\mathrm{q}_{21}$ & 0.083508 & $\mathrm{q} \mathrm{C}_{19}$ & -0.028 \\
\hline$\underline{\mathbf{q} \mathbf{N}_{23}}$ & $-\underline{0.277703}$ & $\mathrm{q} \mathrm{C}_{20}$ & -0.234 \\
\hline $\mathrm{q}_{25}$ & 0.027240 & $\mathrm{qC}_{21}$ & -0.022 \\
\hline $\mathrm{q} \mathrm{C}_{27}$ & 0.322425 & $\mathrm{q}_{22}$ & -0.237 \\
\hline $\mathrm{q}_{28}$ & -0.145945 & & \\
\hline $\mathrm{q} \mathrm{O}_{29}$ & -0.356745 & & \\
\hline $\mathrm{q}_{30}$ & -0.290921 & & \\
\hline $\mathrm{q} \mathrm{C}_{32}$ & -0.213261 & & \\
\hline $\mathrm{q} \mathrm{C}_{33}$ & -0.234911 & & \\
\hline $\mathrm{q}_{40}$ & -0.065233 & & \\
\hline
\end{tabular}


Table 3. Geometric parameters of penicillin acid and DGD.

\begin{tabular}{|c|c|c|c|}
\hline Parameter & Penicillin acid & Parameter & DGD \\
\hline $\mathrm{r}\left(\mathrm{C}_{1}-\mathrm{C}_{2}\right), \mathrm{nm}$ & 0.139 & $\mathrm{r}\left(\mathrm{C}_{1}-\mathrm{O}_{2}\right), \mathrm{nm}$ & 0.121 \\
\hline $\mathrm{r}\left(\mathrm{C}_{1}-\mathrm{H}_{8}\right), \mathrm{nm}$ & 0.109 & $\mathrm{r}\left(\mathrm{C}_{1}-\mathrm{H}_{8}\right), \mathrm{nm}$ & 0.110 \\
\hline $\mathrm{r}\left(\mathrm{C}_{5}-\mathrm{C}_{11}\right), \mathrm{nm}$ & 0.149 & $\mathrm{r}\left(\mathrm{C}_{1}-\mathrm{C}_{3}\right), \mathrm{nm}$ & 0.147 \\
\hline $\mathrm{r}\left(\mathrm{C}_{11}-\mathrm{C}_{14}\right), \mathrm{nm}$ & 0.149 & $\mathrm{r}\left(\mathrm{C}_{3}-\mathrm{C}_{4}\right), \mathrm{nm}$ & 0.134 \\
\hline $\mathrm{r}\left(\mathrm{C}_{14}-\mathrm{N}_{16}\right), \mathrm{nm}$ & 0.131 & $\mathrm{r}\left(\mathrm{C}_{4}-\mathrm{C}_{5}\right), \mathrm{nm}$ & 0.145 \\
\hline $\mathrm{r}\left(\mathrm{N}_{16}-\mathrm{C}_{18}\right), \mathrm{nm}$ & 0.144 & $\mathrm{r}\left(\mathrm{C}_{5}-\mathrm{C}_{6}\right), \mathrm{nm}$ & 0.136 \\
\hline $\mathrm{r}\left(\mathrm{C}_{14}-\mathrm{O}_{17}\right), \mathrm{nm}$ & 0.139 & $\mathrm{r}\left(\mathrm{C}_{6}-\mathrm{N}_{7}\right), \mathrm{nm}$ & 0.139 \\
\hline $\mathrm{r}\left(\mathrm{O}_{17}-\mathrm{C}_{19}\right), \mathrm{nm}$ & 0,139 & $\mathrm{r}\left(\mathrm{N}_{7}-\mathrm{S}_{13}\right), \mathrm{nm}$ & 0.178 \\
\hline $\mathrm{r}\left(\mathrm{C}_{19}-\mathrm{O}_{20}\right), \mathrm{nm}$ & 0.121 & $\mathrm{r}\left(\mathrm{S}_{13}-\mathrm{C}_{14}\right), \mathrm{nm}$ & 0.139 \\
\hline $\mathrm{r}\left(\mathrm{C}_{19}-\mathrm{C}_{18}\right), \mathrm{nm}$ & 0.146 & $\mathrm{r}\left(\mathrm{C}_{14}-\mathrm{N}_{15}\right), \mathrm{nm}$ & 0.116 \\
\hline $\mathrm{r}\left(\mathrm{C}_{18}-\mathrm{C}_{21}\right), \mathrm{nm}$ & 0.136 & $\mathrm{r}\left(\mathrm{C}_{5}-\mathrm{C}_{16}\right), \mathrm{nm}$ & 0.147 \\
\hline $\mathrm{r}\left(\mathrm{C}_{21}-\mathrm{H}_{22}\right), \mathrm{nm}$ & 0.110 & $\mathrm{r}\left(\mathrm{C}_{16}-\mathrm{O}_{17}\right), \mathrm{nm}$ & 0.125 \\
\hline$\left.\underline{\mathrm{r}\left(\mathrm{C}_{21}\right.}-\mathrm{N}_{23}\right), \mathrm{nm}$ & $\underline{0.139}$ & $\mathrm{r}\left(\mathrm{C}_{16}-\mathrm{N}_{18}\right), \mathrm{nm}$ & 0.141 \\
\hline$\left.\underline{\mathrm{r}\left(\mathrm{N}_{23}\right.} \underline{-} \underline{\mathrm{H}_{24}}\right), \mathrm{nm}$ & $\underline{0.099}$ & $\mathrm{r}\left(\mathrm{N}_{18}-\mathrm{C}_{21}\right), \mathrm{nm}$ & 0.149 \\
\hline $\mathrm{r}\left(\mathrm{N}_{23}-\mathrm{C}_{25}\right), \mathrm{nm}$ & 0.148 & $\mathrm{r}\left(\mathrm{C}_{21}-\mathrm{C}_{22}\right), \mathrm{nm}$ & 0.151 \\
\hline $\mathrm{r}\left(\mathrm{C}_{25}-\mathrm{C}_{27}\right), \mathrm{nm}$ & 0.153 & $\mathrm{r}\left(\mathrm{N}_{18}-\mathrm{C}_{19}\right), \mathrm{nm}$ & 0.149 \\
\hline $\mathrm{r}\left(\mathrm{C}_{27}-\mathrm{O}_{29}\right), \mathrm{nm}$ & 0.121 & $\mathrm{r}\left(\mathrm{C}_{19}-\mathrm{C}_{20}\right), \mathrm{nm}$ & 0.151 \\
\hline $\mathrm{r}\left(\mathrm{C}_{27}-\mathrm{O}_{30}\right), \mathrm{nm}$ & 0.135 & & \\
\hline r (O30-H31), nm & 0.095 & & \\
\hline r (C25-C28), nm & 0.155 & & \\
\hline r (C28-S40), nm & 0.186 & & \\
\hline r (S40-H41), nm & 0.131 & & \\
\hline r (C28-C32), nm & 0.152 & & \\
\hline r (C28-C33), nm & 0.152 & & \\
\hline
\end{tabular}

\section{Conclusions}

The obtained semi-empirical computer modelling calculations can be used to explain the mechanism of the formation reaction of the cyanine dye, and this fact is a novelty of this scientific work. Besides, the defined geometric and electronic parameters of cyanine dye molecules confirm the chemical structure of a given molecule.

Effect of substituents on the electronic density distribution in molecules of penicillin acid and DGD, evaluated by electronic effects-inductive and mesomeric and the electronic density distribution in the molecules of these compounds, explained by using semiempirical AM1 calculations, proves once again that a negatively charged nitrogen atom of penicillin acid molecule can attack the positively charged carbon atom in a strongly polarized carbonyl group of DGD.

Thus, the explanation of the mechanism of this reaction by theoretical possibilities of quantum-chemical method AM1 was confirmed by interpretation of experimental data by methods of spectral analysis (IR, NMR, NMR-spectroscopy) [19], to identify features of the chemistry of the reaction formation of cyanine dyes.

This scientific workout in perspective is a further study of the reaction by other methods in quantum chemistry. 


\section{References}

[1] Korenman, I.M. (1975) Photometric Analysis. Methods for the Determination of Organic Compounds. 2nd Edition, Rev. and Add.-Moscow: Chemistry, 359 p.

[2] Polyudek Fabini R. and Beirikch T. (1981) Organic Analysis: A Guide to the Analysis of Organic Compounds, including Drugs. Substances / Tr. from German, Leningrad: Chemistry, 624 p.

[3] Beisenbekov, A.S. and Aliyev, A.M. (1986) Application of Formation Reactions of Polymethine Dyes in Pharmaceutical Analysis. Alma-Ata, 95 p.

[4] Kramarenko, V.F. and Popova, V.I. (1972) Photometry in Pharmaceutical Analysis. Health, Kiev, 191 p.

[5] Traven, V.F. (2004) Organic Chemistry: A Textbook for High Schools. 2 Volumes/VF Traven.-M.: ICC “Akademkniga”, $582 \mathrm{p}$.

[6] Sykes, P. (1991) Reaction Mechanisms in Organic Chemistry. 4th Edition, Tr. from English.-M.: Chemistry, 447 p.

[7] Beisenbekov, A.S., Zhubayeva, R.A. and Boshkayeva, A.K. (1993) Photometric Determination of Anabazin with Fenilmethilpirazolonom Hydrochloride by Reaction Formation of Polymethine Dyes: Method. Instructions/S.D. Asfendyairov ASMI-Almaty, 12.

[8] Arzamastsev, A.P. (2004) Pharmaceutical Chemistry: Textbook. 3rd Edition, Rev.-M.: GEOTAR Media, 640 p.

[9] Belikov, B.G. (2007) Pharmaceutical Chemistry. In 2 Parts: Textbook/V. G. Belikov, 4th Edition, Rev. and Add.-M.: MED Press Inform., 624 p.

[10] Arzamastsev, A.P. (2008) Pharmaceutical Chemistry: Textbook. 3rd Edition, Rev.- M.: GEOTAR Media, 640 p.

[11] Belikov, V.G. (2008) Pharmaceutical Chemistry: Textbook. 2nd Edition, MED Press Inform, M., 616 p.

[12] Aksenova, E.N., Andrianova, O.P., Arzamastsev, A.P., et al. (2001) Guide to the Laboratory Lessons in Pharmaceutical Chemistry. M.: Medicine, 384 p.

[13] Boshkayeva, A.K. and Kamilov, Kh.M. (2009) Application in the Analysis of the Reaction Formation of Cyanine Dyes. Pharmaceutical Journal, No. 2, 55-59.

[14] Boshkayeva, A.K., Omarova, R.A. and Beisenbekov, A.S. (2009) Assessment of the Quality of the Cyanine Dye of Betalaktamid by NMR- and H-NMR Spectroscopy. Bulletin of KazNMU, No. 2, 55-59.

[15] Burstein, K.Ya. and Shorygin, P.P. (1989) Quantum Chemical Calculationsin Organic Chemistry and Molecular Spectroscopy. Nauka, Moscow, $104 \mathrm{p}$.

[16] Clark, T. (1990) Computer Chemistry. Mir, Moscow, 381 p.

[17] Stepanov, N.F. (2001) Quantum Mechanics and Quantum Chemistry. Theoretical Foundations of Chemistry. Mir, Moscow, 519 p.

[18] Boshkayeva, A.K. (2009) The Study by IR Spectroscopy, NMR and 13 C NMR Spectroscopy in Cyanine Dye-Lactamide. Pharmaceutical Journal, No. 1, 50-53.

[19] Boshkayeva, A.K. (2009) The Chemistry of the Formation of Cyanine Dyes. Scientific Review, No. 3, 4-7. 
Scientific Research Publishing (SCIRP) is one of the largest Open Access journal publishers. It is currently publishing more than 200 open access, online, peer-reviewed journals covering a wide range of academic disciplines. SCIRP serves the worldwide academic communities and contributes to the progress and application of science with its publication.

Other selected journals from SCIRP are listed as below. Submit your manuscript to us via either submit@scirp.org or Online Submission Portal.
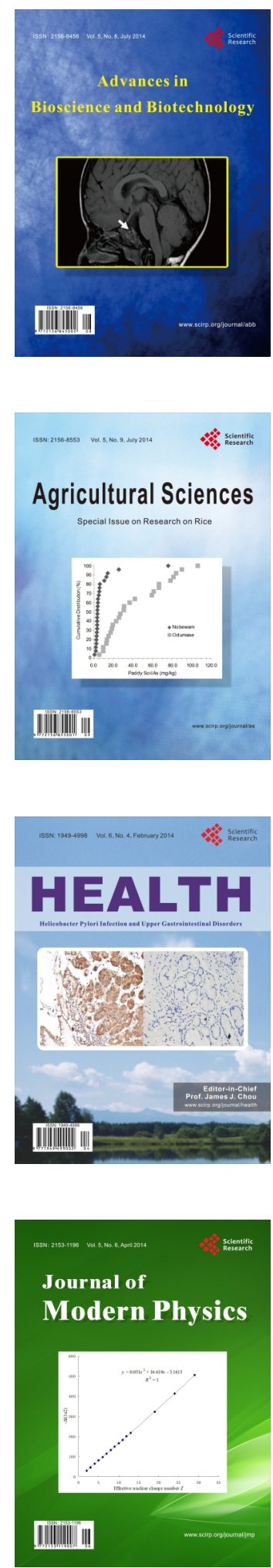
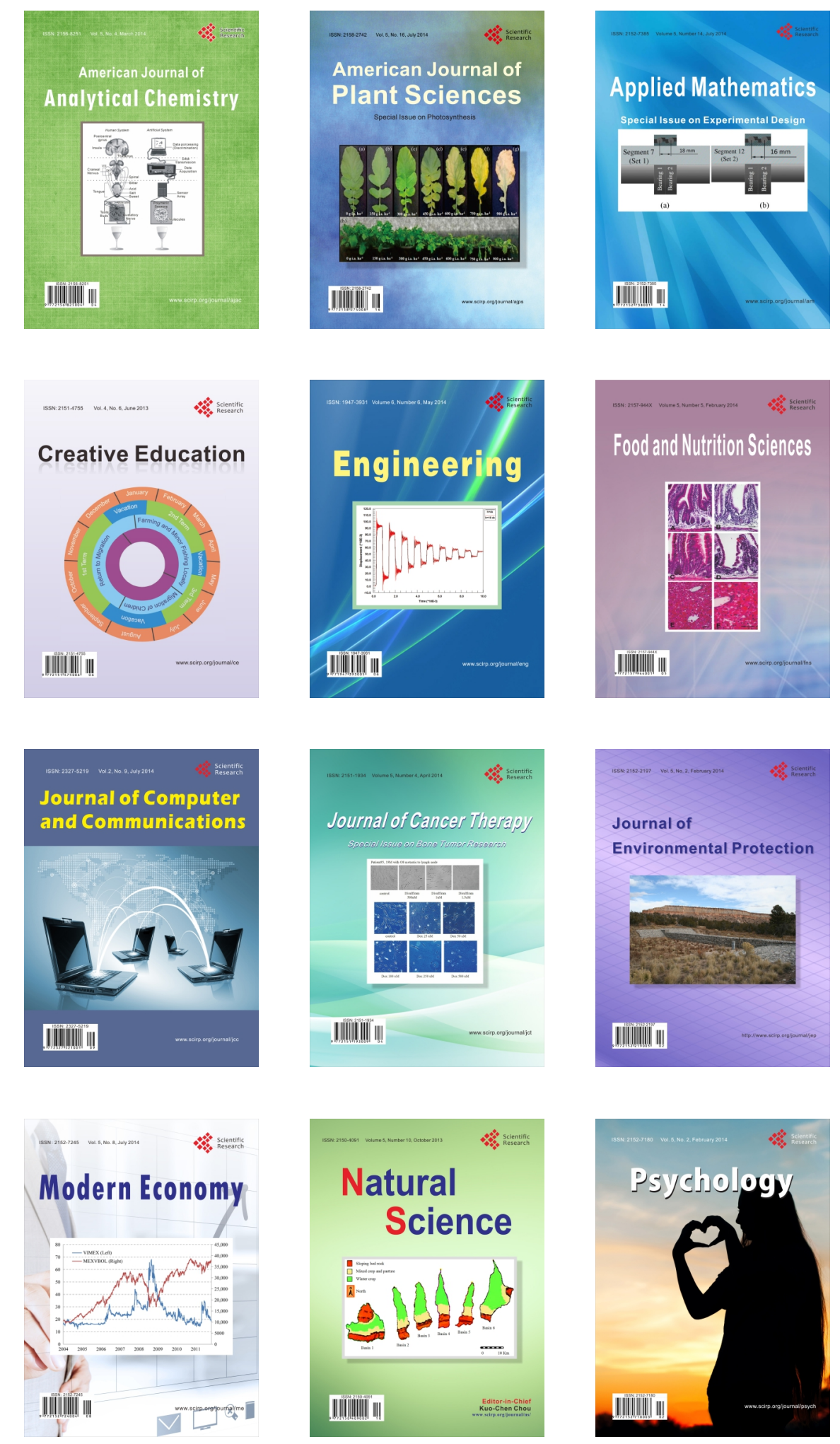